\title{
Germanica
}

\section{Helmut Koopmann, Thomas Mann. Studien, statt einer}

Biographie

Königshausen \& Neumann, 2016, 571 p.

\section{Carola Hähnel-Mesnard}

\section{OpenEdition}

\section{Journals}

Édition électronique

URL : http://journals.openedition.org/germanica/3884

DOI : 10.4000/germanica.3884

ISSN : 2107-0784

\section{Éditeur}

Université de Lille

\section{Édition imprimée}

Date de publication : 30 juin 2017

Pagination : 205-207

ISBN : 9782913857391

ISSN : 0984-2632

\section{Référence électronique}

Carola Hähnel-Mesnard, « Helmut Koopmann, Thomas Mann. Studien, statt einer Biographie»,

Germanica [En ligne], 60 | 2017, mis en ligne le 30 juin 2017, consulté le 08 janvier 2021. URL : http:// journals.openedition.org/germanica/3884; DOI : https://doi.org/10.4000/germanica.3884 


\section{Comptes rendus de lecture}

Sarah Schmidt (Hrsg.), Sprachen des Sammelns. Literatur als Medium und Reflexionsform des Sammelns, Paderborn, Wilhelm Fink Verlag, 2016, $729 \mathrm{~S}$.

Sammeln gehört zu den menschlichen Tätigkeiten, die eng mit dem Zivilisationsprozess einerseits und der Ausbildung von Individualität und Identität andererseits zusammenhängen. Zwar führt kein gradliniger Weg von den Jägern und Sammlern der Vorzeit zu den fürstlichen Wunderkammern der Frühen Neuzeit und den Bibliotheken, Archiven und Museen der Moderne, aber bei dem Impuls, Dinge zu sammeln, sich mit ihnen zu umgeben und sie für die Nachwelt aufzubewahren, scheint es sich um eine universelle anthropologische Konstante zu handeln. Nicht zufällig rücken das Sammeln und die Sammlung just in dem Augenblick in den Fokus verschiedener Wissenschaftsdisziplinen, in dem die Grenzen traditionellen Sammelns offenkundig werden und sich zugleich mit den digitalen Speichermedien neue Formen des Sammelns etablieren.

Die Literaturwissenschaft ist aufgrund ihres Gegenstandes und ihrer Fragestellungen mit der Problematik des Sammelns in besonderer Weise verbunden. Man denke nur an Goethes subtile Überlegungen in Der Sammler und die Seinigen (1799), die manischen Sammlertypen in Stifters und Canettis Texten, W. G. Sebalds melancholische Poetik des Sammelns oder das monumentale Echolot-Projekt (1993-2005) von Walter Kempowski. Die ersten Anstöße kamen jedoch nicht von der Literaturwissenschaft, sondern von den Kulturwissenschaften. Erinnert sei hier an Aby Warburgs Mnemosyne-Atlas (posthum 2000), von dem wichtige Anregungen für interdisziplinäre Debatten ausgegangen sind. 
In diesen Debatten ist auch das Forschungsprojekt „Sprachen des Sammelns" verortet, das von 2010 bis 2014 von der Deutschen Forschungsgemeinschaft gefördert worden ist und dessen Ergebnisse in dem gleichnamigen Sammelband Sprachen des Sammelns (2016) nun vorgelegt werden. Zugleich profitieren Projekt und Band von Entwicklungen, die unter dem Schlagwort material turn seit den 1990er Jahren diskutiert werden und die Aufmerksamkeit auf die Materialität und Medialität von kulturellen und literarischen Prozessen gelenkt und den Blick für wissens- und institutionsgeschichtliche Zusammenhänge sowie für genderspezifische und epistemologische Aspekte geschärft haben. Eine Reihe von Tagungen, Ausstellungen sowie die Einrichtung eines Masterstudiengangs „Sammlungsbezogene Wissens- und Kulturgeschichte" an der Universität Erfurt zeigen, dass sich hier ein Forschungsfeld formiert hat, das die Literaturwissenschaft produktiv mit anderen Disziplinen vernetzt und zur Attraktivitätssteigerung des Faches beitragen kann.

Dass die Ergebnisse des Forschungsprojekts „Sprachen des Sammelns" in einem Sammelband vorgelegt werden, ist konsequent, stellt aber eine besondere Herausforderung dar: Wie soll man einen Band von über 700 Seiten und 44 Beiträgen in einer notgedrungen beschränkten Rezension angemessen würdigen? Und wie soll man damit umgehen, dass der Band nicht nur fachwissenschaftliche Aufsätze, sondern auch Arbeiten und Statements von Künstler/innen enthält, die integraler Bestandteil des Forschungsprojektes waren? Hilfreich bei der Orientierung erweist sich ein Blick in die Einleitung und die Gliederung des Bandes. Die vier großen Kapitel des Buches

\section{Die Beschreibbarkeit der Dinge und die Dinglichkeit der Sprache}

(S. 31-195)

II. Dynamik und Ordnung der Sammlung (S. 199-311)

III. Taxonomien des Menschen - Archive des Humanen (S. 315-495)

IV. Die „Unterseite“ der Sammlung (S. 499-657)

fragen nach dem Was, dem Wie, dem Wann und dem Warum des Sammelns und nach den Kriterien der Auswahl und des Ausschlusses von Dingen. Sie basieren auf den verschiedenen Arbeitsgruppen des Projekts und werden jeweils mit einem einführenden Beitrag der Projektleiterinnen eröffnet. Leitende Gesichtspunkte der einzelnen Beiträge sind dabei zwei Annahmen: Erstens, dass es eine „Analogie zwischen Sprache und Sammlung“ (S. 16) gibt, und zweitens, dass „literarische Praktiken und Thematisierungen des Sammelns häufig einen implizit oder explizit geführten poetologischen Diskurs mitführen" (S. 21). Auch wenn nicht alle Beiträge des Bandes gleichermaßen intensiv auf diese Hypothesen Bezug nehmen, so stellen sie doch - ungeachtet 
ihrer Unterschiedlichkeit - spannende Fallstudien zu eben diesen beiden Grundannahmen dar.

Mit der Behauptung einer engen Verbindung zwischen Sprache, Sammeln und Poetologie lenkt der Band den Blick auf Zusammenhänge, die in den gegenwärtigen, stark um Gedächtnis, Erinnerung und Vergessen kreisenden Debatten über die Bedeutung des Sammelns bislang eher unterrepräsentiert waren. Das mag auch einer der Gründe sein, warum die Deutsche Forschungsgemeinschaft das Projekt finanziert hat. Hierbei hat sie auf ein interessantes neues Förderungsformat gesetzt, das zwischen großen Verbundprojekten und kleineren Einzelvorhaben angesiedelt ist und die Vernetzung von Wissenschaftler/innen aus unterschiedlichen Bereichen, Alters- und Statusgruppen zum Ziel hat. Zum engeren Netzwerk gehörten Christine Blättler, Judith Kasper, Susanne Komfort-Hein, Mona Körte, Bärbel Küster, Armin Schäfer, Susanne Scholz und Ulrike Vedder, die zugleich für die einzelnen Kapitel des Bandes zuständig sind.

In fünf Workshops unter alternierender Leitung und einer Reihe von mittleren und kleineren Arbeitstreffen sowie im Austausch mit Künstler/innen auf einer Tagung auf Schloss Solitude in Württemberg und einer zweiten im Künstlerhaus Schloss Wiepersdorf wurde die Fragestellung nach der Verbindung zwischen Sprache und Sammeln bearbeitet. Literatur wird als ein Medium verstanden, in dem ,über das Sammeln, über Sammler und Sammelinstitutionen reflektiert wird“, und zugleich „mit sprachlichen und genuin literarischen, poetischen oder rhetorischen Ordnungssystemen und Textverfahren Sammlungen erstellt werden“ (S. 17): „Sammelnde Schreibweisen und die motivische Auseinandersetzung mit dem Sammeln bedingen sich dabei wechselseitig, sie bilden sich indessen keineswegs eins zu eins aufeinander ab.“ (Ebd.) Ein interessantes, etwas isoliert stehendes Beispiel stellt in diesem Zusammenhang der Publikationstyp „Gesammelte Werke“ dar, mit dem sich Philip Ajouri am Beispiel von Kellers und Goethes Werken beschäftigt. Untersuchungen etwa zu den Sammeltätigkeiten der Brüder Grimm oder anderen Sammlungstypen hätten diesen unterrepräsentierten germanistischen Aspekt verstärken können. Es ist jedoch müßig, auf Lücken aufmerksam zu machen. Produktiver ist es, sich mit dem zu beschäftigen, was Aufnahme in den Band gefunden hat.

So greift das dritte Kapitel ,Taxonomien des Menschen - Archive des Humanen" mit seiner Orientierung an physiognomischen, medizinischen, juristischen, kolonialen und rassistischen Praktiken entschieden über den engeren literarischen Bereich hinaus. Hier wird der Mensch selbst zum Objekt des Sammelns mit dem Ziel, ein Wissen vom Menschen zu vermitteln, das gesellschaftlich und politisch im Sinne des Foucault'schen Machtdispositivs eingesetzt werden kann. Dabei spielt die Literatur eine nicht zu unterschätzende Rolle, stellt sie doch 
die Muster und Metaphern zur Verfügung, nach denen kategorisiert und gewertet wird. Zugleich ist die Verbindung zwischen Texten und Bildern in diesen Fällen besonders eng. Das gilt auch für die künstlerischen Arbeiten von Jacqueline Baum und Ursula Jakob, Bernd Behr, Maria Hanl, Regina Hilber, Matthias Megyeri, George Steinmann, Sue Waterman und Heidemarie von Wedel, die jeweils von Mitgliedern des Netzwerkes in eigenen Beiträgen gewürdigt werden.

Zeitlich umfassen die Aufsätze eine Spanne von der Frühen Neuzeit bis in die Gegenwart und haben einen deutlichen Schwerpunkt im 19. und 20. Jahrhundert, wobei auch Beispiele aus der englischen, französischen und portugiesischen Literatur vertreten sind. Der Band versammelt Studien aus der Literaturwissenschaft und Philosophie sowie den Kunst- und Kulturwissenschaften. Dazu kommen Beiträge von Schriftstellerinnen, Künstlern und Künstlerinnen, die den interdisziplinären Anspruch des Projekts unterstreichen. Manche Autoren und Themen werden Kenner/innen schmerzlich vermissen, sie werden aber entschädigt durch die Fülle der Aspekte und die geschickte Kombination zwischen Überblicksartikeln und Einzelinterpretationen. Insgesamt handelt es sich um einen Band, der theoretisch und argumentativ auf einem hohen Niveau operiert, auch wenn die Auswahl im Einzelnen nicht immer zwingend erscheint. Einige Autor/innen sind - gemäß ihrer Beteiligung an dem Projekt - mit zwei oder drei Beiträgen vertreten.

Für die Rezensentin waren - abgesehen von den informativen Einleitungen der Projektleiterinnen in die vier Kapitel - diejenigen Beiträge am spannendsten, die sich deutlich an den theoretischen Vorgaben des Vorhabens orientieren und jeweils eine dezidierte Fragestellung oder einen spezifischen Text oder Autor/in ins Zentrum ihrer Argumentation stellen. $\mathrm{Zu}$ nennen sind hier „Gendered objects. Literarische Ding- und Geschlechtercodierungen“" von Ulrike Vedder, „Das wandernde Taschentuch - Herta Müllers widerständige Sammlung“ von Katja Schubert, , Der nicht aufgehende Rest - zum Widerstreit zwischen Objekt und Ding in der Moderne" von Dominik Finkelde, „Vom Ding zum Zeichen: Abc-Bücher und Buchstabensuppen“ von Mona Körte, „Vom Ausscheren und Einsammeln der Buchstaben. Saussures Anagramm-Studien und Freuds Fehlleistungen“ von Judith Kasper, „Sammelpunkte“ von Michael Niehaus, „Sammeln ohne Zugriff: Sammeln ohne Sinn! Über den zunehmenden Verlust hypermedialer Wissensräume im Zeitalter ihrer elektronischen Speicherbarkeit" von Harald Kraemer, „Ordnungsversuche im Gebiet des Wahnsinns: Archive, Akten, Biographien“ von Armin Schäfer, „Existenzen sammeln - Existenzen schreiben: Überlegungen zu M. Foucault, W. Kempowski und F. Hoppe“ von Sarah Schmidt, „Im Zweifel für die Reste: Lumpensammler und andere Archivisten der Moderne“ von Barbara Thums, „Aufgesparte gummiringe nie benutzte griffel'“. 
Nicht-sterbliche Überreste in der Gegenwartsliteratur" von Gisela Ecker und last, not least „Fremdeigene Wortreste - Sprache als Sammlung in Herta Müllers Collagen“ von Sarah Schmidt, der Herausgeberin des Bandes.

Das ist zugegebenermaßen eine kleine und sehr subjektive Auswahl aus einer Sammlung von mehr als vierzig Beiträgen. Allein die Titel zeigen jedoch, wie vielfältig die Fragestellungen und wie unterschiedlich die Herangehensweisen an das Generalthema sind. Andere Leser und Leserinnen mögen andere Schwerpunkte setzen. Für all diejenigen, die sich für Dinge und Objekte, Sammlungen, Sammler und Sammeln interessieren, ist der Band in Gänze ein unverzichtbares Kompendium, in dem sich - wie in jeder Sammlung - Anregungen für die eigene Arbeit finden und Entdeckungen für weitere Forschungen machen lassen. Ein umfassendes Literaturverzeichnis und ein sorgfältiges Personenregister erleichtern die Orientierung in dem voluminösen Buch, das durch die zahlreichen Bildbeilagen einen zusätzlichen visuellen Reiz besitzt.

Inge Stephan

Bettina Thiers, Experimentelle Poetik als Engagement. Konkrete Poesie, visuelle Poesie, Lautdichtung und experimentelles Hörspiel im deutschsprachigen Raum von 1945 bis 1970, Hildesheim, Georg Olms Verlag, 2016, 614 p.

Le présent ouvrage est la version publiée d'une thèse de doctorat soutenue en 2014. Bettina Thiers y propose un panorama de la poésie dite expérimentale dans sa phase la plus productive, des années 1950 aux années 1970. On y retrouve les figures centrales de cette création littéraire comme Eugen Gomringer, Ernst Jandl, Gerhard Rühm, Helmut Heißenbüttel, mais aussi des auteurs moins étudiés, et pourtant très importants pour le développement et la diffusion de cette poésie, tels Max Bense ou Reinhard Döhl.

Alors que la critique a souvent opposé cette production tournée vers la forme à la littérature engagée au contenu explicitement politique, Bettina Thiers veut montrer qu'il existe bel et bien une dimension politique de la poésie concrète, visuelle et sonore ainsi que des pièces radiophoniques expérimentales et en dégage les spécificités. L'engagement se révèle, selon elle, dans la poétique des textes, d'où le titre de l'ouvrage. Il ne s'agit cependant ni de « la posture destructrice des avant-gardes historiques » (p. 483), ni d'une littérature engagée telle que définie par Sartre. En dehors de quelques textes qui traitent d'événements politiques comme le célèbre poème de Jandl wien: heldenplatz, la portée politique de la poésie expérimentale se manifesterait avant tout dans la conception d'une littérature qui ébranle les modes de pensée et les certitudes du lecteur, lui-même partie prenante de la construction du sens. Comme 
l'écrit Bettina Thiers en conclusion : «Letztendlich geht es in der experimentellen Poesie also weniger um das 'Engagement' ihrer Autoren denn um die Emanzipation des Lesers von konventionellen Denkmustern als Voraussetzung für sein potentielles Engagement. Darin liegt ihre politisch subversive Wirkungskraft » (p. 483). On le comprend ici, Bettina Thiers associe une analyse précise de la poésie expérimentale à une problématisation de la notion d'engagement.

L'ouvrage, composé de six parties, se caractérise par sa démarche méthodique, qui entraîne certes parfois quelques répétitions, mais garantit une grande clarté dans la démonstration. L'auteure s'appuie sur des références théoriques pertinentes, présentées en début de partie ou de sous-parties, et sur des analyses minutieuses des textes expérimentaux, reproduits en couleurs avec des manuscrits et tapuscrits dans une annexe fort à propos pour ce type de littérature. L'ensemble est complété par une bibliographie détaillée, qui aurait toutefois pu faire une distinction entre les sources et la littérature critique.

La première partie constitue l'introduction. Elle rappelle la tension entre engagement et littérature expérimentale et pose la problématique d'un « engagement par l'effet esthétique » (p. 18), en s'appuyant sur la pensée de Jacques Rancière, avant de délimiter le corpus par une présentation des auteurs abordés (Max Bense, Helmut Heißenbüttel, Reinhard Döhl, Claus Bremer, Franz Mon, Gerhard Rühm, Ernst Jandl, Eugen Gomringer) et d'évaluer l'état de la recherche. L'ampleur du corpus conduit, dans la suite de l'ouvrage, à des choix lorsqu'il s'agit d'apporter des exemples. Ces choix sont toujours pertinents car ils renvoient à l'auteur ou aux auteurs le(s) plus représentatif(s) pour la démonstration, mais le lecteur aurait été intéressé, dans certains cas, par une approche plus globale, présentant la position de tous les auteurs du corpus, par exemple dans la sous-partie sur la subjectivité, tout en étant conscient que cette option n'était pas possible dans les limites d'un seul volume. La deuxième partie se penche sur la poésie expérimentale, en dessine les contours en distinguant la poésie concrète de la poésie visuelle et en revenant sur les caractéristiques de la pièce radiophonique expérimentale. Elle s'attache également à montrer les différences par rapport aux avant-gardes historiques et à situer la production expérimentale d'aprèsguerre dans son ou plutôt ses contextes : allemand, autrichien et suisse. Enfin, elle s'interroge sur le rapport des auteurs expérimentaux à la notion d'engagement et de non-conformisme. La troisième partie traite de la critique du langage comme critique socioculturelle. Elle revient tout d'abord sur l'influence de Mauthner, mais surtout de Wittgenstein, souvent cité par les poètes expérimentaux, puis sur l'apport de Derrida et de Foucault. Plusieurs pages sont consacrées à la déconstruction du discours comme critique idéologique dans l'œuvre de Helmut Heißenbüttel, également au centre des réflexions sur la place du sujet 
dans la poésie expérimentale (sujet face au collectif et face à l'histoire). Dans la quatrième partie, il est question du lien entre les nouvelles technologies et la création expérimentale. Dans ce contexte, l'étude porte sur les œuvres de Gomringer, qui considère que la poésie doit accompagner la technicisation du monde (p. 279) et de Max Bense, un des premiers auteurs de textes générés par ordinateur, ainsi que sur la poésie visuelle de Franz Mon et les pièces radiophoniques de Heißenbüttel, qui renouvellent radicalement le genre. L'intérêt des écrivains expérimentaux pour les évolutions technologiques de leur époque est une preuve supplémentaire de leur ancrage dans la société dans laquelle ils vivent. On est donc loin de l'image souvent véhiculée par la critique des poètes détachés de la cité. La cinquième partie met en relation les notions de globalisation et de démocratie avec la poésie expérimentale. Elle relève alors les connexions internationales entre les poètes expérimentaux et envisage une acception positive du phénomène de globalisation qui ne serait pas synonyme de nivellement, mais de dynamisation de la culture, notamment telle qu'elle apparaît dans la poésie plurilingue de Ernst Jandl (« Mischsprachen »). De plus, le rôle imparti au lecteur apparenterait cette poésie à des pratiques de l'actionnisme et de l'art de la performance ainsi qu'aux réflexions sur la démocratie participative qui se développent dans les années 1970. Bettina Thiers fournit ici une analyse inédite de la performance de Jandl lors de sa lecture au Royal Albert Hall de Londres, en analysant la voix et la gestuelle de l'auteur ainsi que l'interaction qui se crée avec le public. Enfin, la sixième partie est la conclusion de l'ouvrage, qui rapproche la poésie expérimentale de la théorie de «l'œuvre ouverte » d'Umberto Eco et situe cette poésie par rapport à la postmodernité.

L'ampleur et l'apport du travail de Bettina Thiers sont donc indéniables, et on ne peut qu'inviter toutes les personnes qui s'intéressent à la littérature engagée et à la poésie expérimentale ou qui veulent la découvrir, à lire ce livre qui est incontestablement un ouvrage de référence en la matière.

Aurélie Le Née

Jean-Pierre Chassagne (dir.), L'auctorialité à travers les mises en scène de l'auteur et de l'artiste dans le monde germanique. Autorschaft in den Inszenierungen des Autors und des Künstlers im deutschsprachigen Raum, Publications de l'Université de Saint-Étienne, coll. « Voix d'ailleurs », 2016, 185 p.

Partant du constat d'un « retour de l'auteur » et du postulat que « tout acte littéraire s'accompagne d'une certaine théâtralité » (J.-P. Chassagne) dont le but est à la fois de provoquer l'adhésion du public et de situer l'écrivain dans l'arène littéraire (soumise aux fluctuations 
idéologiques et aux impératifs du marché), les contributions réunies dans cette publication s'inscrivent dans le prolongement des travaux entamés il y a une dizaine d'années en Allemagne par Gunter E. Grimm et Christian Schärf autour de la notion de mise en scène interne - par le biais de postures littéraires adoptées par l'écrivain - ou externe - par la critique, le monde de l'édition, les médias - de l'auteur. «Sans masque, le moi n'est rien » : telle est la thèse défendue par C. Schärf et illustrée dans sa préface à travers une lecture originale du Voyage dans le Harz en hiver de Goethe. À travers différentes études de cas visant, ainsi que le mentionne J.-P. Chassagne, à " exemplifier la théorie », et dans un élargissement de la réflexion aux champs musical et artistique, l'ouvrage propose quatre angles d'approche de la notion d'auctorialité.

Le premier chapitre s'intéresse à la fictionnalisation de la biographie de l'écrivain ou de l'artiste : à la mise en scène du personnage de Goethe à travers des témoins extérieurs ; à une esthétisation de sa propre vie, savamment orchestrée par Wagner, afin d'emporter l'adhésion d'un public de disciples et d'assurer sa gloire posthume ; et, à l'inverse, à la remise en question par Schnitzler de l'autorité et de l'instance auctoriale dans la farce Au Grand Guignol. En marge de toute fiction, le second volet analyse différentes stratégies de construction de l'image auctoriale dont l'enjeu est la difficile conquête de la notoriété (dans le cas de Hohl ou de Sebestyén), la préservation de la « personne » par l'effacement de l'image privée au profit de la seule mise en scène de l'acte d'écriture (Mayröcker) ou l'affirmation de la souveraineté de l'auteur (Bernhard). Dans le troisième volet, c'est cette construction même qui fait l'objet d'un questionnement : si Thomas Mann se fait volontiers le complice de la construction de son image médiatique invitant à lire son œuvre, conformément au désir des lecteurs de l'époque, comme le «paratexte de [sa] vie », le récepteur se trouve à l'inverse déstabilisé par la mise en question du pacte autobiographique (chez Éric Chevillard et Felicitas Hoppe) et par le détournement de l'autoportrait de sa fonction première (chez l'artiste Martin Kippenberger). La dernière partie, enfin, analyse la façon dont se constitue l'image de l'auteur par le jeu de l'intertextualité : dans un dialogue qui s'instaure avec un modèle (celui qu'entretient Thomas Bernhard avec Montaigne, qui fait de lui-même son propre sujet d'étude, exemple par excellence de la souveraineté à laquelle aspire l'écrivain autrichien), ou dans un recours et des références à l'héritage littéraire germanique ou aux textes fondateurs (chez Beer-Hofmann revêtant la figure auctoriale d'une dimension messianique, ou chez Wolfskehl afin de se positionner dans le champ littéraire au lendemain d'Auschwitz).

À une époque caractérisée par une demande, voire une exigence, croissante d'authenticité et de transparence, cet ouvrage peut paraître à maints égards intempestif - et c'est bien ce qui lui confère toute 
sa richesse, son intérêt et son potentiel de subversion. Le « retour de l'auteur » se fait en effet sous le signe de la complexité, en marge des repères fournis par la littérature biographique qui connaît un formidable regain d'intérêt et par l'hagiographie. La notion même d'authenticité devient paradoxale (en ce qu'elle serait, elle aussi, telle est déjà la grande leçon de Montaigne, le résultat d'une mise en scène), tandis que se pose invariablement la question, à laquelle aucune réponse catégorique ne saurait être donnée, de l'adéquation entre l'image officielle de l'auteur propagée - avec ou sans sa complicité - par les médias et au service de la promotion de l'œuvre, la représentation de l'auctorialité que se fait l'écrivain ou l'artiste lui-même, et les attentes du public, également fondées sur une idée de la littérature et de l'art. Difficile de dire où commence et où s'arrête la théâtralité et si quelque chose ou non, dans cette entité qu'est le créateur, échappe à la mise en scène qualifiée par C. Schärf d'« énergie ", une énergie qui entraîne le lecteur et contribue à son plaisir dans son improbable conquête de l'auteur qui se refuse à toute saisie définitive.

Andrée Lerousseau

Helmut Koopmann, Thomas Mann. Studien, statt einer Biographie, Würzburg, Königshausen \& Neumann, 2016, 571 p.

Avec ce volume réunissant une trentaine d'articles, Helmut Koopmann, le doyen des recherches sur Thomas Mann en Allemagne, souhaite rendre accessibles à un public plus large des conférences, essais, communications de colloques ou autres textes publiés de manière éparse, écrits entre 1990 et 2014. Il s'agit d'études qui questionnent et approfondissent tel ou tel aspect de l'œuvre de Thomas Mann, tentent d'établir des liens et des correspondances entre différents textes de l'œuvre, et privilégient, selon le chercheur, une approche moins inspirée par les «théories modernes » que par le contexte culturel et historique dans lequel les textes s'inscrivent.

Éditeur, entre autres, d'une encyclopédie de référence sur la vie et l'œuvre de Thomas Mann et auteur d'une étude remarquée sur les liens entre les frères Heinrich et Thomas Mann, Koopmann ne souhaite pas livrer, sur le tard, «sa » biographie de l'auteur. Néanmoins, il n'ordonne pas ses différentes contributions dans l'ordre de leur première parution, mais dans l'ordre chronologique de la vie de Thomas Mann, souhaitant faire émerger de ce «puzzle » le «profil spirituel » de Mann autrement que dans une biographie traditionnelle.

Les deux premières parties du volume sont consacrées à l'œuvre de jeunesse, elles sont particulièrement importantes car elles éclairent les débuts d'une écriture et d'une œuvre qui se développera par la suite tout en gardant certaines caractéristiques - au niveau du style et des thèmes 
- mises en place très tôt. Koopmann s'interroge ainsi sur l'impact de l'œuvre de Goethe qui dépasse très tôt la simple lecture scolaire et se ressent dès les premières nouvelles, comme dans Déchue (Gefallen, 1894) ou dans Paillasse (Der Bajazzo, 1897) : une lecture possible en relève la proximité avec l'histoire de Marianne dans Wilhelm Meister pour la première, avec Werther pour la seconde. Plus loin, dans un chapitre entièrement dédié à l'influence de Goethe, Koopmann relève également les traces goethéennes dans La Mort à Venise, avant de s'interroger sur sa présence dans l'œuvre plus tardive. Concernant les interrogations et thématiques du jeune Thomas Mann, on trouve une étude sur la signification de la maladie : la description du typhus dans Buddenbrooks se lit comme un condensé métaphorique du déclin de la famille, et la présence du thème de la « dégénérescence » dans de nombreuses nouvelles de la même époque est interprétée à travers le prisme d'un essai de Max Nordau.

On connaît l'importance que revêt le mythe chez Thomas Mann depuis l'écriture de La Mort à Venise. Pour Koopmann, la nouvelle devient le point de départ d'une interrogation sur le rôle des mythes dans l'œuvre de jeunesse des frères Mann et sur leur rupture avec les pratiques littéraires du XIX ${ }^{\mathrm{e}}$ siècle : Heinrich Mann aussi bien que son frère recourent désormais au mythe pour rendre compte du caractère double et ambigu de la réalité ambiante, tout en démasquant le mythe, en le démystifiant. Un autre article s'intéresse au rapport de Thomas Mann au XIXe siècle, siècle qui l'a vu naître et dont il porte les traces, mais avec lequel il entretient une relation contradictoire. Parmi les problématiques ressenties comme ambiguës en cette fin du XIXe siècle, on trouve celle de l'artiste qui est évidemment au cœur de l'œuvre de jeunesse et qui traduit un questionnement personnel du jeune Thomas Mann. Koopmann s'y intéresse à l'aune des réserves exprimées par d'autres auteurs et penseurs de l'époque qui situent l'artiste dans une filiation avec des marginaux redoutables tels que le criminel, l'imposteur ou l'aventurier, puis revient sur l'influence de Wagner, vu à travers la lecture de Nietzsche, qui déclenche chez Thomas Mann une réflexion sur lui-même et sur son statut d'artiste.

D'autres chapitres rassemblent des articles à la fois de contextualisation ou d'étude de détails consacrés aux grandes œuvres romanesques : La Montagne magique (1924), Joseph et ses frères (1933-1943) et Le Docteur Faustus (1947). Plusieurs articles élucident ensuite les relations de Thomas Mann avec d'autres écrivains et intellectuels de son époque, tout d'abord avec son frère Heinrich, mais également avec son fidèle éditeur Samuel Fischer ou encore les relations avec Franz Werfel et Bertolt Brecht en exil. Le volume se clôt sur des thèmes plus intimes, comme le rapport de Mann à la religion ou à la mort. 
Il est impossible de rendre compte ici de toute la richesse des thématiques abordées, mais le lecteur intéressé par l'œuvre de Mann trouvera sans aucun doute des éléments nouveaux ou peu connus dans ce recueil d'articles précis et savants, agréables à lire, qui prennent position et tiennent compte des débats de la vaste littérature critique tout en y apportant une touche personnelle.

\section{Carola Hähnel-Mesnard}

Daniel Argelès, Klaus Schlesinger ou l'écriture de l'histoire - Berlin et l'Allemagne, 1937-2001, Villeneuve d'Ascq, Presses Universitaires du Septentrion, 2017, $354 \mathrm{p}$.

En proposant la première monographie en français de Klaus Schlesinger, Daniel Argelès nous invite, par une analyse fine et exigeante de quelque 15 romans et nouvelles, à revisiter 60 ans d'histoire de l'Allemagne et de l'Allemagne en Europe. Cette étude retrace ainsi l'évolution de la production littéraire d'un écrivain allemand qui aura connu l'Allemagne national-socialiste, l'Allemagne de la capitulation, l'Allemagne divisée en quatre zones d'occupation, la RDA (avant et après la construction du Mur), la RFA des années 1980, et enfin l'Allemagne réunifiée. L'ouvrage est divisé en quatre grandes périodes, qui mettent chacune l'accent sur des enjeux thématiques et d'écriture précis. Les années 1960 montrent la confrontation avec le passé national-socialiste et ses implications dans le contexte est-allemand, notamment le travail d'introspection éludé. Les années 1970 thématisent l'interrogation du présent socialiste et de son histoire récente, le quotidien de la RDA (avec une approche courageuse de nombreux sujets alors tabous), la question de l'épanouissement de l'individu dans cette société. Les années 1980 marquent l'épreuve du déracinement avec le passage à l'Ouest (avec des interrogations sur la notion d'« exil »), l'appropriation de cette autre Allemagne et de l'univers ouest-berlinois, l'intégration dans le mouvement alternatif (avec les déceptions face aux anciens soixante-huitards) et une évolution dans le regard posé sur l'histoire allemande et la possibilité de dépasser le clivage Est/Ouest. Les années 1990 amènent un retour sur la complexité de l'expérience historique de l'unification et finalement le bilan d'une vie. Avec ces quatre décennies, c'est l'expérience des « métamorphoses de l'Allemagne » (p. 15) après la Deuxième Guerre mondiale qui est au cœur du propos. On ne cessera d'ailleurs de s'étonner, à la lecture de cette monographie, de constater combien Klaus Schlesinger fut dans son époque : «Une petite enfance sous le 'III Reich', marquée par la fascination pour le Führer, une adolescence dans le Berlin en ruines de l'après-guerre, placée sous le signe de la défaite, de la disparition de son père au front et du choc de la découverte des crimes nazis, la vie d'apprenti puis de laborantin dans la ville 
divisée mais encore ouverte des années 1950, le choc de la fermeture des frontières en août 1961, la vie à l'ombre du Mur et dans le socialisme 'réellement existant', la quête des marges de manœuvre littéraires, politiques et personnelles jusqu'au durcissement du régime communiste autour de l'affaire Biermann en 1976, l'exil à l'Ouest dans le sillage de son exclusion de l'Union des écrivains de RDA en 1979, l'expérience du Berlin-Ouest alternatif des années 1980 (en particulier le mouvement des squatters), la surprise et les désorientations de la chute du Mur et de la réunification, la question des compromissions pendant le socialisme, avec en particulier la découverte des 2000 pages du dossier de surveillance monté contre lui et son entourage par la Stasi, la question du double passé allemand et de l'avenir de la nouvelle Allemagne » (p. 16).

L'ouvrage analyse l'œuvre littéraire de Schlesinger sur l'histoire et le destin des Allemands, leur rôle, leurs choix, leurs responsabilités, comme Allemands dans la Seconde Guerre mondiale, dans le socialisme réel, dans les divisions de la Guerre froide, dans les recompositions politiques et identitaires de l'unification. Par touches répétées, Daniel Argelès replace Klaus Schlesinger dans le contexte littéraire et politique tout au long de ces quatre décennies de travail. L'ouvrage interroge l'évolution de l'écriture de Schlesinger pour dire cette histoire allemande des 70 dernières années, avec la conviction du lien étroit entre fiction et histoire (Paul Ricœur), pour dire l'engagement de leur auteur dans cette histoire, pour dire aussi le cheminement personnel, la construction de soi : la fiction est le lieu de recomposition et de construction de soi dans et face à l'histoire, l'histoire est le lieu de la confrontation et de l'émancipation à la fois esthétique, éthique et politique. Les enjeux de forme sont étudiés avec attention, leur évolution bien montrée, le vocabulaire utilisé est interrogé avec finesse. Au centre de son propos, Daniel Argelès place la notion de « liminalité », particulièrement bienvenue et qui prend tout son sens au fur et à mesure que le propos se déploie.

Même si le choix est hautement subjectif, on pourra retenir de ces déambulations et analyses littéraires nourries par une connaissance personnelle de l'auteur, plusieurs fois rencontré à Berlin, différents textes. Tout d'abord la première nouvelle, publiée en 1960, David, où Klaus Schlesinger imagine les derniers instants de plus jeunes des insurgés du ghetto de Varsovie, jeune messager entre deux groupes de résistance du ghetto capturé par les Allemands. Klaus Schlesinger questionne dans son texte d'une part le travail de mémoire est-allemand sur ce que l'on n'appelait pas encore la Shoah et d'autre part le travail d'introspection sur les crimes nazis, le rôle des pères et le sentiment de culpabilité des fils. De la production des années 70, on attirera l'attention sur deux nouvelles parues en 1977, Die Spaltung des Erwin Racholl (1977), plongée dans un univers doublement kafkaïen et stalinien, et Am 
Ende der Jugend, qui raconte la fin de matinée et l'après-midi du 13 août 1961 et le passage à l'Est de l'ami du narrateur, Martin: comme l'écrit très justement Daniel Argelès, cette nouvelle « ramène un événement emblématique de la Guerre froide, de la division allemande, de l'histoire de la RDA et de Berlin à un [...] bout de rue [...] synecdoque spatiale, temporelle et existentielle extrêmement intense » (p. 140) - un texte de l'appréhension (ou non) par l'individu de l'événement historique en train de se produire, de l'instant fatidique où tout bascule. Des années 80 , on pourra retenir le roman Matulla und Busch, premier texte publié après ce que Günter Kunert avait nommé le « Platzwechsel », le passage à l'Ouest et qui montre la rencontre improbable entre un duo de retraités ouest-allemands et des squatteurs à Berlin-Ouest, belle tentative de Schlesinger de s'approprier l'espace littéraire, géographique et politique de Berlin-Ouest.

Tentative pleinement réussie de sortir Klaus Schlesinger d'un injuste oubli, ce travail en appelle finalement un autre, différent mais complémentaire, afin de permettre au public français de goûter au plaisir de cette lecture : celui de traduire cette œuvre, dont seul un roman (Matulla und Busch, traduit en 1990 par Jean-Marie Argelès), une nouvelle (Am Ende der Jugend/La fin de la jeunesse, traduite en 2003 par Jean-Marie Argelès) et des extraits du recueil Leben im Winter/La vie en hiver (traduits en 2002 par Nicole Taubes) ont été traduits. 
